\title{
The Treatment of Fibromyalgia Using Vibrational Herbal Medicine and the Laws of Ancient Wisdom
}

\author{
Almoez LeDin Ellah M. S. Eltouny ${ }^{*}$ \\ The National Center for Research, Egypt \\ "Corresponding Author: Dr. Almoez LeDin Ellah M. S. Eltouny, The National Center for Research, Egypt, Tel: \\ 0020-2-359-2083; E-mail: eltounya@ hotmail.com
}

Received: 20 October 2018; Accepted: 08 November 2018; Published: 28 November 2018

\begin{abstract}
This is a case study to determine a treatment and/ or a cure for Fibromyalgia. This was a study and trial on one patient who had been diagnosed with Fibromyalgia in the USA. She had been under medication for Fibromyalgia, but that did not appear to have made a great deal of difference and the patient could control her pain or condition. My work dealt with finding treatments for unusual ailments that could not be cured by conventional methods. The technique of Vibrational Herbal Medicine was discovered by accident, while experimenting with the measurement of Herbs to find out their energy qualities. Our research continued for a few years and the results were published in "Explore Magazine" published in Prescott, Arizona in 2000. We studied what energies affect Blood Pressure, and concentrated heavily on finding a treatment or cure, for Hep. C viral, which was endemic in Egypt, at that time. Our research continued and spread to many other ailments and diseases over the next twenty years. In 2017, we were approached by an acquaintance who was aware of our work and wanted us to take the case of this female patient with Fibromyalgia. It was an intriguing case as I had never treated a similar case. The following is a base on which we came to the technique employed in Vibrational herbal Medicine.
\end{abstract}

Keywords: Fibromyalgia; Vibrational Herbal Medicine; Blood Pressure

\section{Introduction}

The Ancient Chinese discovered that ailments of the human body are the result of an increase or a decrease in the vital energies flowing in the energy paths of that body. Their objective became to bring these energies into balance 
in order to heal or cure the specific ailment. They knew that the energy had to be manipulated to become more or less, depending on the ailment. There was one basic failure; they could not measure the increase or decrease of that energy. They did not appear to have a measuring device to do that. Around that time, or probably earlier, the Ancient Egyptians made the same discovery, however, they had a device to measure the changes occurring in the level of energy they were measuring. These energies are unseen energies flowing through the body and affecting every single part or organ of that body. The device that they made was the Universal Pendulum. This pendulum, and other shapes and forms of it are seen until today on the walls of the temples in Kom Ombo, Edfu and other temples still standing in Egypt. There is a whole section of Pendulum Exhibits in the Louvre in Paris. This Pendulum in the hands of a practiced Adept or Practitioner, can open inroads in discovering the ailments in the human body or "see" the hidden flow of energy through the human body and the changes that occur. All our studies are conducted using a Pendulum and using the technique I devised to measure the changes of energy due to changes in the condition of the patient under study. This Pendulum can measure the energy of a virus unseen by modern science and its effects and the effects of a medicine on that virus or ailment, measuring the increase or decrease of the energy associated with that virus or ailment. The procedure used may not conform to scientific research parameters used in research, but this is a totally different science and will produce significant and plausible results. It has helped in measuring the results of new treatments, without actual testing using theoretical testing, since it will measure the changes of the energy of the medicine as it reacts with the energy of the specimen, without the patient ingesting any of it. One may even choose among several chemical medicines for the same ailment in order to obtain the best effective treatment.

\section{Description}

This herbal approach is based on ordinary herbs mixed together in fixed proportions in specific herbal mixtures that are designed to heal a certain ailment. Although the mixtures are totally herbal, the effects of the herbs are not determined by the physical and chemical properties of the herbs in the mixtures but on the vibrational properties of the herbs. This technique was discovered by accident, and may have been used by the Ancient Egyptians and the Ancient Chinese.

We have been able to discover the underlying rules governing the usage of herbs in Vibrational Herbal Medicine. These rules and regulations were published as a paper called "The Rules of Ancient Wisdom and Herbal Medicine." The important fact to consider is that these mixtures are basically safe herbal mixtures that actually affect the patient positively, eliminating the effect of the illness or reducing its effects appreciably, without any side effects to mention.

\section{Utilization}

This technique, through the ability to measure the vibrational energy of organs of the body, and the changes occurring in them also allows the practitioner to be able to recognize the presence of viruses and to be able to measure their energy level changes, i.e., the changes brought about by the herbal medicine taken by the patient, on the energy of the virus and on the patient. 
This approach has helped in formulating herbal mixtures that are designed to attack specific viruses, gradually eliminating the virus and curing the patient. These mixtures are patentable and are protected under international copyright laws. Over the past twenty years we have been able to study and prepare treatments and cures for several lethal ailments, even some that have been discovered to be viral in origin. Our objective in this case study is to determine the cause and treatment of Fibromyalgia.

\section{Description of Fibromyalgia}

The Mayo Clinic publication " Health Letter" describes Fibromyalgia as: Fibromyalgia is a disorder characterized by widespread musculoskeletal pain accompanied by fatigue, sleep, memory and mood issues. Researchers believe that fibromyalgia amplifies painful sensations by affecting the way your brain processes pain signals. Symptoms sometimes begin after a physical trauma, surgery, infection or significant psychological stress. In other cases, symptoms gradually accumulate over time with no single triggering event. Women are more likely to develop fibromyalgia than are men. Many people who have fibromyalgia also have tension headaches, temporomandibular joint (TMJ) disorders, irritable bowel syndrome, anxiety and depression. While there is no cure for fibromyalgia until now, a variety of medications can help control symptoms. Exercise, relaxation and stress-reduction measures also may help.

\section{Application of VHM to the treatment of Fibromyalgia}

In Mid March 2017, a patient diagnosed in the USA, with having Fibromyalgia, approached me to see if it were possible to treat Fibromyalgia using my herbal techniques. We had not treated anyone previously with that disease and we had to check up on it to see what the possibilities were. We met with the patient and listened to what her description of her ordeal was. Since her diagnosis was made in the US, it probably was reliable and accurate since the symptoms would easily be confused with other ailments. Our first approach, as was customary, was to check to find out the actual energy of the muscles of the body in this patient. We used a measurement technique that we devised, to measure the energies of the muscles. This technique is based on the Chinese energy nomenclature and our own design [1].

Energy of the Muscles of the patient on $15^{\text {th }}$ of March 2017.

$Q=-30$

$\mathrm{Yn}=-44$

$\mathrm{Yg}=-44$

Omega $=-20$

As a comparison, the normal energy characteristics of the muscles of a healthy female of the same age; would be:

$\mathrm{Q}=90$

$\mathrm{Yn}=90$ 
$\mathrm{Yg}=90$

Omega $=36$

These results indicated that whatever the reason of the illness, it affected the energy of the muscles and reduced it from the normal levels of a healthy body, giving rise to the symptoms and what we call Fibromyalgia. After the first examination, we discovered that the ailment was not caused by a virus since we could not determine the energy signature of any virus. That meant that a different cause affected the muscles and reduced the energy to the level that caused the ailment. The method of treatment was to design an herbal mixture, based on Vibrational Herbal treatment theories and to test it on the patient, to normalize the energy of the muscles using a mixture of ordinary herbs that resonated with the muscles of the body and enhanced its energy, without any adverse side effects. The main concept was to normalize the energy of the muscles and that would eliminate the cause of the ailment.

Our design yielded a special mixture, made from several herbs that reacted well with the patient and did not have any adverse side effects in our simulation testing on the patient. This mixture enhanced the level of energy of the muscles towards the normal range. This mixture was called Mix Muscle Cure sp \# 7. This mixture was made according to the design parameters, mixed and blended then encapsulated in 900mg gelatin capsules. The objective of this mixture was to enhance the energy of the muscles of the whole body and bring it up to normal levels. The dosage for the first treatment was two 900mg capsules once a day after a meal, but at least 30 minutes from any other oral medication. The duration of the treatment was 25 days. Another condition was not to stop whatever medication she was taking at that time. She was asked to report any adverse side effects she encountered. No problem occurred. The duration of the treatment was 25 days and she started on the $17^{\text {th }}$ of March 2017 . We measured her progress using our techniques and did not hear from her of any side effects.

After the period of treatment, we found that the new energy characteristic levels of the muscles were as follows:

$\mathrm{Q}=8$

$\mathrm{Yn}=22$

$\mathrm{Yg}=20$

Omega $=5$

This was a positive change from the first reading and indicated a small change in the level of energy. This change however, was mildly registered by the patient, by a small reduction in the level of pain experienced, and did not seem to have any effect on the symptoms. She indicated that the pain dissipated more easily than before with pain medication. In our theoretical testing, we discovered that the Mixture Muscle Cure sp\#7 was no longer effective and would not change the outcome if taken for an additional period. A new mixture was needed to continue her progress. It had to be more effective and more powerful. After several trials, a new mixture fitting the requirements was designed, with the same resonance as her body and reacted very well in the theoretical trials. This was called Sp. 
Mixture no.9, to be taken 2 capsules, 900mg each, after a meal and half an hour from any other medication. The treatment duration was 20 days and started on $15^{\text {th }}$ of May 2017.

In the follow up of the treatment, the patient was responding positively and the energy characteristics of the muscles improved. The improvement was not as much as we had hoped, but the energy levels were rising. Since there were no records of using such a method for the treatment of Fibromyalgia, our only references were those of the patient and how she felt. The latest levels of the Muscle energies were as follows:

$\mathrm{Q}=25$

$\mathrm{Yn}=32$

$\mathrm{Yg}=32$

Omega $=10$.

These values were in comparison to the normal values of a female of the same age but not suffering from Fibromyalgia, and these were:

$\mathrm{Q}=90$

$\mathrm{Yn}=90$

$\mathrm{Yg}=90$

Omega $=36$.

We still had a long way to go, but the energy levels were improving, the patient was slightly better on the whole, than previously noted. Mixture \#9 worked well, but was not up to our expectations. We started some trials for a new and improved mixture to continue what we had started and that was safe to the patient and resonated with her frequency. We designed Sp. Mixture no 3. The theoretical tests showed that it worked well with the patient and would improve the energy levels of the muscles. The patient was given 15 days of treatment in the form of two 900mg gel capsules to be taken after a meal and half an hour from any other medication.

After the term of the last treatment special mixture 3, we checked the energy levels and they were as follows:

$\mathrm{Q}=54$

$\mathrm{Yn}=55$

$\mathrm{Yg}=55$

Omega $=20$.

These were a marked improvement over the previous levels and should translate into something positive for the patient. She mentioned that she felt better, not as tired, with her stamina levels higher and better than she had felt, and that she had stopped one of her regular medicines, a prescription drug, due to its unavailability in the market at that time. She was candid that the withdrawal from that prescription drug caused some agony but she endured. This 
was a very positive step and indicated that the results obtained from our theoretical testing actually indicated the changes occurring in the patient and a reduction of the effect of the ailment on the patient. The special mixture \#3 did not totally cure the patient and we needed another mixture to complete the healing process and that would be the one mixture to take for any new patient. This whole process is that of trial and error. There were no precedence to our treatment and we had no reference except the condition of the patient. Trials on a new mixture were started and finally reached the design of Special Mixture \#4that would be the one mixture to take to complete the healing of Fibromyalgia in any patient, or as a whole, since it would work from an early state. This treatment took into account all the previous treatments and their results. The trials indicated that the patient needed only twelve days to complete her cure. This dosage was made and given to the patient with its instructions. At this time the patient was more confident since her condition improved and her stamina was greatly enhanced.

This new mixture worked quite well and the energy characteristics of the muscles improved, so did the condition of the patient. She felt well, the feeling of tiredness diminished totally, and morning swelling of the muscles disappeared.

The energy Characteristics of the muscles on $18^{\text {th }}$ of August 2017 was as follows:

$\mathrm{Q}=90$

$\mathrm{Yn}=95$

$\mathrm{Yg}=92$

Omega $=28$

These were the same figures measured in Mid December 2017, at the writing of this research report. Even today, in the late summer of 2018, the patient is fine embarking on her life's journey with vigor and optimism. There had not been any relapse in her condition for several months, even after discontinuation of the Herbal Mixture and her condition appeared normal. She felt a huge difference between the initial treatment and the final one. She felt as a normal person would. The patient traveled to the US during the Christmas Holidays, but was unable to schedule an appointment with her previous Physician, so there was not any new description of her condition since his initial diagnosis. Upon her return, she felt tired for a couple of days, with some swelling in the hands, also for a couple of days, then her condition became normal again. This meant that none of the old symptoms returned and that she rebounded from any physical changes. However, our latest measurements of the muscle energy characteristics, indicated exactly the same readings as Mid December. . Even today, in the late summer of 2018, the patient is fine embarking on her life's journey with vigor and optimism.

\section{Conclusion}

Our conclusion was that there was no relapse and she is now as cured as could be. We must note that the value of Omega, the well being indicator in vibrational Herbal Medicine, shows that there was some effects of the ailment that were not eliminated. However, the steady condition of the muscles, indicate a level of cure with no relapse. 
From the above, it may be that we have reached a reasonably safe and effective way to treat Fibromyalgia by using oral capsules, designed by using the techniques of Vibrational Herbal Medicine and with totally herbal ingredients.

\section{References}

1. The Laws of Ancient Wisdom" Eltouny, Almoez, Explore Magazine, Prescott, Arizona.

Citation: Almoez LeDin Ellah M. S. Eltouny. The Treatment of Fibromyalgia Using Vibrational Herbal Medicine and the Laws of Ancient Wisdom. Archives of Clinical and Medical Case Reports 2 (2018): 254-260.

(C) 9 BY
Creative Commons Attribution (CC-BY) license 4.0 